\title{
APRENDIZAJE SIGNIFICATIVO Y EDUCACIÓN AMBIENTAL: ANÁLISIS DE LOS RESULTADOS DE UNA PRÁCTICA FUNDAMENTADA TEÓRICAMENTE
}

\author{
Gur uceaga, Arant zazu ${ }^{1}$ y Gonzál ez García, Fer mín M.² \\ ${ }^{1}$ Natur Zientziak Mintegia. San Fermín Ikastola. Pamplona \\ ${ }^{2}$ Departamento de Psicología y Pedagogía. Universidad Pública de Navarra
}

\begin{abstract}
Resumen. Se presenta en este artículo una aplicación de la teoría de Ausubel, Novak y Gowin al ámbito de la educación ambiental, concretada en el tema de la desertificación. Se pretende mostrar la utilidad de este marco teórico y de los instrumentos emanados de él, el mapa conceptual y el diagrama u ve, para favorecer un aprendizaje significativo en alumno/as de $3^{\circ}$ de ESO de un centro concertado de la comarca de Pamplona. El estudio muestra que el diseño e implementación de un módulo instruccional sobre la desertificación, basado en este marco teórico, produce unos resultados en el alumnado del grupo experimental, indicadores de un aprendizaje más significativo y de un cambio más positivo en sus actitudes, en relación con esta problemática medioambiental, en marcado contraste con los resultados obtenidos en un grupo de control.

Palabras clave. Educación ambiental, desertificación, mapa conceptual, diagrama u ve, aprendizaje significativo, módulo instruccional, grupo experimental, grupo control, actitudes.
\end{abstract}

\begin{abstract}
Summary. This article describes the application of the Ausubel, Novak and Gowin theory in an environmental education setting, with desertification as the focal theme. The aim is to demonstrate how this theoretical approach and the concept map and V diagram, which are the tools derived from it, were used to promote meaningful learning among third grade secondary pupils in a state-assisted school in the Pamplona area. The study shows how pupils in a test group, when presented with a teaching module on desertification, designed and implemented according to this theoretical approach, obtained results that were indicative of a more meaningful learning and a positive change of attitude towards this particular environmental problem, in marked contrast to the outcome observed in the control group.

Keywords. Environmental education, desertification, concept map, V diagram, meaningful learning, teaching module, test group, control group, attitudes.
\end{abstract}

\section{CONSIDERACIONES TEÓRICAS PREVIAS}

Las referencias históricas de la educación ambiental (EA) (Estocolmo, 1972; Tblisi, 1977; Moscú, 1987; Río de Janeiro, 1992; Foro Global, 1992; Salónica, 1997) insisten en la idea de que, para lograr los objetivos más relevantes de la misma, es necesario otorgar un papel activo a la persona que aprende en lo que se refiere a su propio proceso de aprendizaje y, como consecuencia de ello, plantea la necesidad de que el papel del profesorado se caracterice fundamentalmente por facilitar las condiciones necesarias (Novo, 1998). Las investigaciones educativas realizadas en el ámbito de la EA, por ejemplo, las citadas en la revis- ta norteamericana Journal of Environmental Education, en diferentes publicaciones de la UNESCO, y en ERIC (Educational Resoruces Information Center), apenas si muestran una realidad en las aulas de educación secundaria en la que se potencie este papel activo de los alumnos y alumnas. De todas formas hay que señalar que existen experiencias relevantes en las que, entre otros, se plantea que la evaluación en la EA tiene que dirigirse fundamentalmente hacia el grado en que el alumnado ha aprendido a comprender la base del problema medioambiental, considerando esta condición como necesaria para favorecer 
unas disposiciones y actuaciones favorables al medio ambiente (Lucas, 1980; Breiting, 1997). Por otro lado, en la medida en que la investigación que presentamos a continuación se sitúa en el área de la ciencias naturales tenemos que considerar también que, en los últimos años y en relación con la enseñanza de las ciencias, existe una investigación fundamentada en varios modelos de enseñanza que nos indica dificultades importantes para lograr que nuestros alumnos y alumnas aprendan ciencia significativamente y desde donde se están realizando interesantes propuestas (Pozo y Gómez Crespo, 1998). La EA se fortalece de estas propuestas tal y como se aprecia en las aportaciones que se realizan desde el modelo de enseñanza basada en la iniciación a la ciencia de la complejidad (García, 1995, 1998). Aunque nuestra propuesta no parte de los mismos presupuestos, pensamos que es importante trabajar en la búsqueda de relaciones entre los diferentes modelos, e incluso en la búsqueda de una integración jerárquica entre los mismos. Además, recientemente Rickinson (2001) realiza un exhaustivo trabajo de revisión crítica de publicaciones relacionadas con el alumnado y el aprendizaje en EA. Su análisis se basó en el detallado estudio de más de cien artículos de revistas especializadas, libros e informes publicados entre 1993 y 1999. Rickinson considera que, en relación con el alumnado y el aprendizaje, las evidencias encontradas, aunque considerables en tamaño, son menos diversas en términos de enfoques teóricos y metodológicos. Además en su estudio se evidencia que los trabajos analizados proporcionan más información acerca de las actitudes y el conocimiento ambiental de los alumnos que de sus preferencias y experiencias educativas, y más acerca de los resultados del aprendizaje que de los procesos de aprendizaje.

Por nuestra parte e intentando perfilar una propuesta de mejora en los procesos de aprendizaje y en los logros de la EA, en esta investigación nos hemos situado con la teoría educativa de Novak (1978) y en su propuesta concreta. Este autor afirma que, para conseguir un aprendizaje más activo y eficaz de los alumnos y para posibilitar que se produzcan en ellos cambios en la comprensión y actitud hacia el medio ambiente, hay que relacionar lo que ya se sabe acerca de la naturaleza del conocimiento y del aprendizaje humano con la EA. Propone, concretamente, relacionar las aportaciones de autores como Toulmin, Khun o Ausubel con la EA. Igualmente plantea que, en la medida en que se facilite el aprendizaje significativo (AS) del alumnado, impulsaría así mismo actitudes más positivas en ellos. De acuerdo con la concepción de educación de Gowin (1981), entendemos el AS como una integración efectiva de pensamiento (conocimiento), sentimientos (actitudes) y acción (conductas), de forma que compartimos plenamente la convicción de que esta integración, tan fundamental para la EA, puede ser facilitada mediante AS. Pensamos que una posibilidad interesante para investigar los logros de la EA en el aula es partir de la concepción global e integradora del AS ya mencionado y, apoyándonos en la concepción ausubeliana del mismo, diseñar e implementar instrucciones que faciliten un aprendizaje más significativo en las aulas. Esta propuesta presenta unos resultados muy positivos de la mano de autores como Brumsted (1990), Stilwell y Brody (1987).

\section{NATURALEZA DEL APRENDIZAJE SIGNIFI- CATIVO}

El logro de un AS es el verdadero fin educativo. Esta propuesta de Novak la comparte también Moreira (1997), que considera el AS como un concepto subyacente a las diferentes propuestas constructuvistas sobre el aprendizaje humano. Añade este mismo autor que el AS planteado en su origen por Ausubel y desarrollado después por Novak y Gowin es uno de los conceptos más útiles para mejorar el aprendizaje escolar.

Desde su perspectiva constructivista, Novak hace hincapié en el concepto de AS tal y como Ausubel lo plantea en su teoría de la asimilación. El AS es la manera natural de aprendizaje de las personas, y los procesos psicológicos que intervienen en el mismo suponen que una estructura cognitiva preexistente del individuo asimila la nueva información. Esta asimilación ocurre en función de las relaciones jerárquicas que el individuo establece entre los conceptos, en las que el concepto más inclusor asimila o subsume otros conceptos más específicos, de manera que, en este proceso, todos los conceptos van adquiriendo un nuevo significado para el individuo. Ausubel propone también que diferenciemos entre el aprendizaje memorístico/mecánico y el significativo, aunque añade que ambos forman parte del mismo continuum del aprendizaje humano. En definitiva, plantea que la naturaleza de las relaciones que el individuo establece con la nueva información es la que condiciona si el proceso de aprendizaje de un individuo concreto está más cercano del aprendizaje memorístico/mecánico o del AS. Cuanto más substanciales sean las relaciones que un individuo establece entre su conocimiento previo y la nueva información que recibe, tanto más significativo será su proceso de aprendizaje; y, por el contrario, cuanto más arbitrarias sean las relaciones que se establecen, más mecánica será la recepción de información y, por consiguiente, el aprendizaje del individuo será más memorístico/ mecánico. Según nuestra perspectiva, lograr un aprendizaje más significativo en relación con unos contenidos de la EA requiere crear en las aulas de secundaria nuevos contextos educativos en los que la posibilidad de acceso a cambios de actitud y comportamiento hacia el medio ambiente sea más factible.

La labor de crear nuevos contextos facilitadores de un aprendizaje más significativo pasa por cumplir unas condiciones mínimas que podemos reducir a tres. En primer lugar el alumno tiene que querer llevar a cabo un proceso de AS. En segundo lugar, para poder establecer unas relaciones significativas y no arbitrarias entre los conceptos, en la estructura cognitiva del alumno deben estar presentes los conceptos más relevantes o inclusores; y por último, para que puedan darse las condiciones del AS es necesario que los materiales de la instrucción escolar sean, en lo que se refiere al significado que se atribuye a los conceptos, lo más transparentes posibles. Para facilitar un aprendizaje escolar más significativo a nuestro alumnado se requiere, además, de instrumentos que ayuden a evidenciar y facilitar este tipo de aprendizaje. Novak, junto con su grupo de investigación, preocupado por conocer el significado que los aprendizajes escolares 
atribuían a determinados conceptos, comenzó utilizando la entrevista clínica piagetiana modificada (Pines, Novak, Posner y Vankirk, 1978) y, en el intento de consensuar dichos significados entre los investigadores implicados, desarrolló un instrumento que verdaderamente responde a aquellos requerimientos: el mapa conceptual (MC). Por otra parte, reconocemos también que la u ve epistemológica de Gowin (González, Novak, 1993, 1996), en la medida que identifica los elementos epistemológicos fundamentales que operan en la construcción de conocimiento, es un instrumento muy válido para ayudar al profesorado en el diseño y elaboración de módulos instruccionales conceptualmente más transparentes.

\section{LOS MAPAS CONCEPTUALES (MMCC)}

Los MMCC, producto de la aplicación de un sólido y coherente marco teórico educativo, son útiles en muchos ámbitos de la educación escolar (Novak, 1997). Por ejemplo, son útiles en la elaboración de los diferentes currículos, en el diseño de módulos instruccionales más lógicos y potencialmente significativos, y también para lograr que los materiales didácticos puedan ser conceptualmente más transparentes. Los MMCC son instrumentos válidos para crear y compartir contenidos de referencia entre profesorado, entre alumnado y profesorado e incluso entre alumnos y alumnas. En esta ocasión nos interesa resaltar que el MC es muy útil para diseñar un módulo instruccional innovador, también para averiguar los conocimientos previos de los alumnos y su evolución en relación con dicha instrucción. Como el MC nos muestra fundamentalmente una imagen esquemática y puntual del conocimiento de un alumno acerca de un tema concreto, puede ser un instrumento que revele el grado de AS llevado a cabo por él. Así en el MC realizado por un alumno se puede detectar, por ejemplo, qué conceptos son los que no utiliza, qué proposiciones erróneas o alternativas realiza y cómo estas proposiciones están estrechamente vinculadas a jerarquías conceptuales no lógicas (González, Morón y Novak, 2001). Así mismo, en un MC se pone de manifiesto claramente cuál es el concepto que un alumno determinado considera más inclusivo y a través de qué diferenciaciones progresivas le atribuye significado. Además, resulta sumamente interesante comprobar con qué facilidad se identifican las relaciones sin jerarquía entre conceptos diferenciados a través de las denominadas reconciliaciones integradoras, las cuales podemos considerar como el aspecto más creativo del AS y de gran potencialidad en relación con el aprendizaje, esencialmente multidisciplinar, de la complejidad en la EA. Con todo ello tendremos la oportunidad de comparar de forma bastante eficaz la estructura cognitiva de dicho alumno con la estructura lógica del conocimiento implicado, y así poder actuar en consecuencia. Hemos de terminar señalando por último que, si los MMCC son instrumentos que pueden reflejar el grado de AS, obviamente también son excelentes instrumentos para identificar el aprendizaje memorístico/mecánico. En la tabla I presentamos los indicadores que pueden utilizarse en un MC para establecer el tipo de aprendizaje.

Tabla I

Indicadores de aprendizaje.

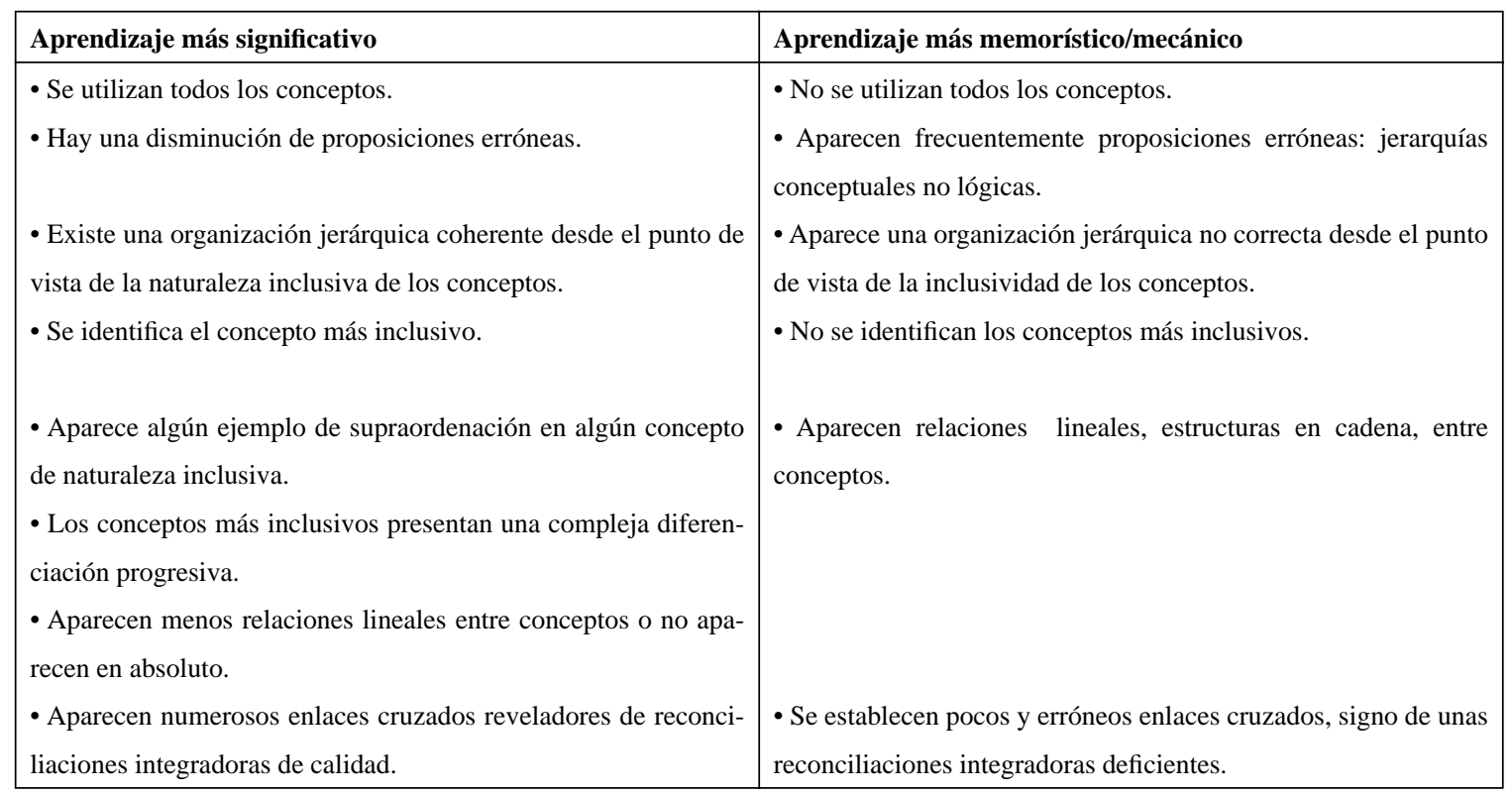


En la investigación objeto de este artículo los MMCC se han utilizado fundamentalmente como instrumento para conocer el grado de AS en un grupo de alumnos y alumnas después de implementar con ellos un módulo instruccional innovador y conceptualmente transparente sobre la problemática medioambiental de la desertificación. En el diseño de la instrucción mencionada también se ha utilizado este instrumento como el referente conceptual a partir del cual se han estructurado las diferentes fases y actividades de enseñanza-aprendizaje.

\section{UVE EPISTEMOLÓGICA DE GOWIN}

El interés de este instrumento ideado por Bob Gowin, colaborador de Novak, radica tanto en su globalidad como en su sencillez (Novak y Gowin, 1988). Sirve fundamentalmente para representar todos los elementos que intervienen en la construcción o examen de un conocimiento. El heurístico en u ve se basa en una concepción constructivista del conocimiento, en la que se reconoce que la búsqueda de nuevo conocimiento y valor asociado a él está íntimamente relacionado con el resto de los elementos de la u ve.

Este instrumento en forma de u ve mantiene diferenciados los cuatro espacios de naturaleza diferente que intervienen, interactuando entre sí, en todo proceso de crear conocimiento. Por una parte, a la izquierda de la u ve tenemos el espacio de aquellos conocimientos filosóficos y teóricos con que se parte a la hora de realizar cualquier tipo de investigación. En el centro de la u ve se explicita aquella cuestión que suscita preocupación y que aflora en relación con lo que se ha plasmado en la parte izquierda de la u ve. En esta parte central se coloca, por lo tanto, lo que llamamos la cuestión central, que es en definitiva la cuestión o problema que impulsa la investigación y que finalmente conducirá al nuevo conocimiento. En el otro extremo, es decir, en el vértice inferior de la u ve, se concretan los hechos y acontecimientos que se quieran realizar para responder a la pregunta central, y los medios y recursos que se utilizarán. En este espacio se incorporan tanto los aspectos y recursos metodológicos como los objetos que se consideran necesarios para llevar a cabo la investigación propiamente dicha. Para terminar nos queda la parte de la derecha de la u ve, donde se transforman los datos resultantes de los registros en la formulación de los juicios de conocimiento y de valor, los que, en definitiva, van a responder directamente a la cuestión central planteada en el centro de la u ve. En nuestro caso, la u ve epistemológica se ha utilizado tanto en el diseño de la investigación objeto de este trabajo como en el diseño de la estructura general de la instrucción innovadora y de las actividades desarrolladas en la misma.

\section{ENTREVISTA PIAGETIANA MODIFICADA}

La entrevista piagetiana modificada sirve fundamentalmente para explorar la estructura cognitiva del alumno concreto, y conocer cuál es el conocimiento idiosincrático y nivel de elaboración que un alumno posee respecto a un tema (Novak y Gowin, 1988; Pines, 1979; Pines, Novak, Posner y Vankirk, 1978; Moreira y Domínguez, 1989). Es decir, la entrevista piagetiana modificada nos informa de lo que un alumno o alumna tiene en su estructura cognitiva, con respecto a un cuerpo de conocimiento. Además hay que resaltar que no sólo se referirá a los contenidos conceptuales y procedimentales, sino que a lo largo de la entrevista también aflorarán con claridad las actitudes y disposiciones del entrevistado. Por lo general, la obtención de información de una entrevista parte, en primer lugar, del análisis proposicional de la transcripción, en la que se separan los discursos independientes que el alumno ha vertido a lo largo de la entrevista. Para conocer lo que un alumno ha aprendido después de un proceso instruccional concreto, podemos diseñar una serie de preguntas y tareas que debe realizar el alumno, en las que éste tenga que utilizar lo aprendido para contestar las preguntas. De esta manera, el análisis proposicional de las entrevistas previa y posterior a la instrucción, nos puede indicar cuál es (cuáles son) el cambio (los cambios) que dicho alumno presenta en referencia a su conocimiento. Así mismo, hay que considerar que cada alumno va a responder de una forma idisioncrática a las preguntas planteadas y que las respuestas que aportan los diferentes entrevistados son de una diversidad muy amplia. Por otra parte, teniendo en cuenta que, con respecto al conocimiento, la humanidad funciona por medio de paradigmas con tendencia a ser globales, es posible que también la población de aprendices y estudiantes presente ciertas comunalidades paradigmáticas. Así, es probable que, entre las diferentes respuestas que un grupo de alumnado aporta en una entrevista piagetiana modificada, encontremos comunalidades que orienten en el proceso de conocer qué es lo que piensan los alumnos y las alumnas en concreto. En definitiva, los resultados del análisis proposicional de las entrevistas nos acerca al conocimiento que un grupo de alumnado mantiene en común y aquellos aspectos del conocimiento que son más idiosincráticos.

En la investigación presente se utiliza la entrevista piagetiana modificada para conocer la evolución de un grupo de alumnos y alumnas sobre lo que sabe en relación con la desertificación y así poder contrastar y complementar esta información con la que nos aportan los MMCC.

\section{PLANEAMIENTO, CONTEXTO Y DESARRO- LLO DE LA INVESTIGACIÓN}

A partir de las consideraciones anteriores, la parte experimental de la presente investigación (Guruceaga, 2001) se realizó en el centro San Fermín Ikastola, centro que escolariza según el modelo lingüístico D (enseñanza en euskera salvo la asignatura de lengua castellana) de la comarca de Pamplona. En concreto, la experiencia se realizó en el curso 1996-97 desde el área de ciencias naturales y en las tres aulas de $3^{\circ}$ de la ESO del centro. En este nivel había un total de 89 alumnos divididos en tres grupos A, B y D; a cada grupo le correspondió una profesora integrante del seminario de ciencias, de las cuales la autora de este trabajo tuvo la responsabilidad de trabajar con el grupo B (grupo experimental) en el que implementó la instrucción innovadora y experimental en relación con la problemá- 
tica medioambiental de la desertificación. Las otras dos profesoras implementaron, en los grupos A y D (grupos control), la instrucción tradicional también en relación con la desertificación, sin referencia a un marco teórico determinado ni a estrategias específicas emanadas de él, como es el caso de la teoría del AS. En la investigación se utilizó un diseño casi experimental, con una organización pretestpostest, en la que se utilizaba la figura del grupo control para detectar la eficacia o la falta de ella de la instrucción experimental (Campbell y Stanley, 1970).

Las hipótesis de trabajo que propusimos en esta investigación fueron dos. En la primera de ellas se postulaba que en los MMCC posteriores se encontrarían más indicadores de AS entre los alumnos que hubieran llevado a cabo la instrucción experimental que, entre los que hubieran participado de la instrucción tradicional. En la segunda hipótesis se sostenía que en los datos obtenidos del análisis proposicional de las entrevistas clínicas modificadas del grupo de alumnado que hubieran desarrollado más eficazmente un AS, se mostrarían actitudes más positivas y posiciones más implicantes en relación con la problemática de la desertificación.

Antes de la implementación del módulo instruccional innovador se llevó a cabo la recogida previa de información acerca de lo que el alumnado sabía sobre la desertificación, utilizándose para ello los MMCC y la entrevista piagetiana modificada. Estos datos se utilizaron en el diseño y la elaboración del propio módulo a partir de la elaboración de un MC sobre la desertificación, que fue consensuado con un experto sobre el tema (Fig.1) y en diseño de actividades didácticas coherentes con los datos proporcionados por el MC y la entrevista inicial. En la preparación de este material se utilizaron tanto MMCC como diagramas u ve. Una vez implementada la instrucción, los alumnos realizaron un $\mathrm{MC}$ final y fueron entrevistados utilizando la técnica de las entrevistas piagetianas modificadas.

Para la realización de las entrevista y el análisis de datos se eligió una muestra representativa desde el punto de vista estadístico. Esa muestra fue el resultado de la elección de 6 alumnos por grupo, aleatoriamente, y por cuotas (representación de tres niveles, con el mismo número de chicas y chicos). Los 89 alumnos realizaron los MMCC previos y posteriores a la instrucción pero, aunque fueron utilizados para su evaluación y calificación escolar habitual, en la investigación propiamente dicha solamente se tuvieron en cuenta los MMCC realizados por los alumnos de la muestra elegida.

Para valorar la significatividad de los resultados de la investigación, el análisis comparativo de los resultados se realizó utilizando la prueba no paramétrica de la probabilidad exacta de Fischer (Calvo, 1994). Esta prueba, que se utiliza en investigaciones de muestras pequeñas e independientes y requiere de tablas de contingencia $2 \times 2$, nos indica que, a menor probabilidad de ocurrencia de algún hecho diferenciador entre los dos grupos, más significativa será dicha diferencia, lo que atribuiremos a la incidencia de la instrucción innovadora. En nuestro caso hemos considerado que la probabilidad de ocurrencia no sobrepase el $3 \%$.

\section{DESARROLLO DE LA INSTRUCCIÓN EN EL GRUPO EXPERIMENTAL}

La desertificación la hemos entendido, tal y como propone la UNEP y el Programa 21 de la Cumbre de Río, como un proceso complejo en el que se interaccionan las actividades humanas y el clima, y cuyo resultado final es la disminución irreversible de la productividad y del valor de los recursos naturales o, dicho de otra manera, la degradación de los ecosistemas terrestres. Se trata de un concepto de gran utilidad, pues resume un mensaje que refleja una dura realidad; pero, al mismo tiempo, ahí reside también su aspecto más problemático, ya que existe el peligro de que la desertificación se identifique únicamente con las condiciones extremas del desierto. Además en relación con esta problemática existe otra dificultad añadida, pues al menos en el entorno lingüístico del castellano se utiliza, junto con el concepto de desertificación, otro concepto de alguna manera similar, el de desertización. En nuestro caso, asumiendo el riesgo de utilizar términos que no están consensuados en relación con su significado, hemos entendido la desertificación como la problemática que subsume la interacción entre procesos climáticos y geológicos con las actividades humanas; y la desertización, en contraste con lo anterior, la hemos entendido como aquel proceso natural que tiende a acentuar la aridez del entorno. Presentamos el MC sobre la desertificación (Fig.1), que fue utilizado tanto como referente para el diseño instruccional como para la evaluación de los MMCC realizados por el alumnado.

En relación con la instrucción, hay que decir fundamentalmente que se ha tenido la intención de acercarse a las condiciones del AS, y que para ello se han utilizado los instrumentos presentados con anterioridad. En concreto, se ha seguido el modelo del LEAP Project (1995), en el cual se distinguen tres fases: introducción, fase de focalización y fase de resumen. En la primera fase, en la introducción, el alumnado dispone de oportunidades para dar su opinión, punto de vista y valoración general acerca del concepto sobre el que se iba a profundizar a lo largo de la instrucción, es decir, acerca de la desertificación. En la segunda fase, se trabaja sobre una serie de reconciliaciones integradoras y conceptos inclusivos, distribuidos en cuatro bloques: proceso de desertización, factores climáticos, erosión, formación de suelo, fertilidad del suelo, actividades o fenómenos humanos como la producción agrícola intensiva y la deforestación. Las reconciliaciones que se trabajan explícitamente en el aula son las que hacen referencia a las consecuencias de la interacción entre fenómenos humanos, como la actividad agrícola intensiva y la deforestación, y un proceso geológico como la desertización. Además, se analiza la relación entre el consumo familiar de productos agrícolas y la producción de los mismos, y se discute en el aula sobre las repercusiones que la pérdida de suelo fértil (degradación del suelo) tiene para nuestro colectivo humano más cercano. Hay que señalar que en esta fase de dar significado a los conceptos más relevantes, por exigencias de la programación anual del área de ciencias naturales, la instrucción experimental se detiene más en los aspectos geológicos que en los aspectos antrópicos. En la fase de resumen, el alumnado, organizado fundamentalmente en grupos pequeños, aplica y reor- 
ganiza lo trabajado a lo largo de la instrucción, teniendo, en definitiva, la oportunidad de realizar propuestas concretas en torno al problema tratado; por ejemplo, a través de la elaboración, en grupo, de un póster de denuncia con propuestas correctoras en relación con la desertificación.

Los medios utilizados en el desarrollo instruccional fueron diversos: textos en relación con los conceptos trabajados, tanto provenientes de fuentes oficiales como de medios de comunicación, libros de texto y elaboración propia. Se utilizaron varios vídeos, entre ellos un programa sobre la desertificación de la serie «Cita con la tierra» de TVE. También se realizaron varias sesiones de trabajo práctico tanto en el entorno del centro como en el laboratorio, en las que se identificaban las características del suelo y se recogían muestras para su posterior análisis en el laboratorio.

\section{ANÁLISIS Y DISCUSIÓN DE LOS RESULTADOS}

La evolución que presentaron los MMCC realizados por el alumnado de ambos grupos, desde el inicio a la finalización de la instrucción, mostraron diferencias significativas. Ante la imposibilidad de presentar todos los mapas, hemos seleccionado algunos representativos de las tendencias generales detectadas en los grupos, como, por ejemplo, los mapas previo y posterior de Mikel, perteneciente al grupo experimental (Fig. 2, 3) y de Elisa, perteneciente al grupo control (Fig. 4, 5).

El primer mapa de Mikel es un mapa estructurado en dos largas cadenas lineales de conceptos que constituyen una estructura típica de un conocimiento arbitrario producto de un aprendizaje mecánico y memorístico. En el segundo mapa se aprecia que Mikel ha identificado el concepto de desertificación como el más inclusivo y, a continuación, éste adquiere significado principalmente a través del concepto erosión, que a su vez presenta una importante diferenciación progresiva. Esta diferenciación que hace Mikel del concepto de erosión en relación con los conceptos de actividad humana y de clima da significado a la desertificación. Además habría que señalar que, en el segundo mapa, Mikel utiliza todos los conceptos, incorporando los que, en el primero, no llegó a utilizar, como por ejemplo salinización, cárcavas y clima mediterráneo. A su vez, presenta menos errores (11 errores en el primer mapa por 4 en el segundo), aunque cabe destacar que persiste el error que supone considerar la desertificación como causa de la «desestructuración del suelo fértil» en lugar de su consecuencia.

Este segundo mapa también muestra más reconciliaciones integradoras entre los conceptos de referencia. Así, relaciona los procesos de desertización, deforestación y las cárcavas con la zona mediterránea; los agentes meteorológicos con la formación de suelo; y la agricultura como posible causante de la deforestación. En definitiva, consideramos que la jerarquía que Mikel establece entre los conceptos se acerca más a su naturaleza lógica y que el conocimiento adquirido por este alumno a lo largo de la instrucción experimental es producto de un proceso de aprendizaje más significativo.

Elisa, al contrario que Mikel, en su primer mapa (Fig. 4) refleja un conocimiento más jerarquizado y diferenciado, no tan lineal, aunque el concepto clima elegido por ella como el concepto más inclusivo presenta una débil diferenciación progresiva. Si analizamos el segundo mapa de Elisa (Fig. 5), veremos que, además de presentar una similitud apreciable con el primer mapa (vuelve a elegir el concepto clima como el más inclusivo), refleja un núcleo conceptual muy confuso en el que se encuentra el concepto desertificación. La confusión se establece en la medida que esta alumna relaciona muchos conceptos entre sí utilizando un sólo enlace y evitando así expresar relaciones más explícitas entre los conceptos afectados.

Hay que añadir además que Elisa no incorporó todos los conceptos al segundo mapa (salinidad, interacción) y reflejó más proposiciones erróneas en este segundo que en el primero ( 6 errores en el primer mapa, 10 errores en el segundo mapa). Presenta los agentes meteorológicos como ejemplo de agentes geológicos, identifica clima mediterráneo con sequía, la agricultura ( su modo de hacer) con degradación de suelo, y las aguas subterráneas como producto del suelo fértil. Añadiremos que esta alumna solamente establece una reconciliación integradora entre las aguas subterráneas y agente geológico externo. Por todo esto podemos concluir que Elisa no mejora en la organización jerárquica establecida entre los conceptos y no ha reflejado un proceso de aprendizaje tan significativo como Mikel.

Los resultados globales del análisis de los MMCC iniciales y finales del grupo muestra son los que se exponen en la tabla II. Se puede afirmar que en los MMCC del grupo experimental, al contrario que en los del grupo control, los conceptos de actividad humana y clima aparecen más diferenciados y reconciliados integradoramente, atribuyendo así significado al concepto desertificación, y reflejan un conocimiento sobre dicho concepto más lógico y más estructurado que los realizados por el grupo que ha aplicado una instrucción más tradicional.

Con relación a los resultados obtenidos del análisis proposicional de las 18 entrevistas realizadas mostramos en las tablas IV y V, en formato original en euskera, fragmentos de las entrevistas realizadas a Mikel y Elisa, que consideramos representativas de la evolución de las tendencias de los dos grupos. Entre las ideas más importantes expresadas por estos alumnos se puede destacar que se percibe que el alumnado del grupo experimental refleja una nueva incorporación significativa de información, como lo pone de manifiesto la incorporación de los conceptos erosión y degradación del suelo en relación con la utilización intensiva del mismo. Este mismo grupo también muestra en su discurso una relación más explícita con la desertificación, ya que se muestra tanto como víctima en la medida que la pérdida de suelo fértil pueda afectar a su propia alimentación, y también como agente, en la medida que es consumidor de productos que provienen de una agricultura intensiva. Debemos añadir que el mismo grupo propone soluciones que pueden afectar a su propio consumo: consumir menos, consumir menos carne, consumir productos de temporada, consumir productos de producción cercana, etc. Los alumnos del grupo control no reflejan, en las entrevistas, una incorporación signifi- 
cativa de nueva información y, en las relaciones que establecen con la problemática medioambiental, no alcanzan el nivel de implicación que muestran los entrevistados del grupo experimental (Tabla III). De todas formas, debemos enfatizar que el significado que los alumnos, en general, atribuyen al concepto desertificación presenta profundos problemas, ya que muchos alumnos no diferencian entre desertificación y proceso de desertización, y atribuyen a esta problemática un significado mediante la utilización de un modelo simplista y catastrofista, relacionado fundamentalmente con una súbita aparición de condiciones desérticas extremas y de deforestación.

Tabla II

Comparación entre resultados de los mapas posteriores.

\begin{tabular}{|c|c|c|c|}
\hline & Grupo control $N=12$ & Grupo experimental $N=6$ & Probabilidad de ocurrencia \\
\hline $\begin{array}{l}\text { La razón entre número de proposiciones correctas y no } \\
\text { correctas ha mejorado }\end{array}$ & 4 & 6 & \multirow[t]{2}{*}{$\mathrm{P}=0{ }^{\prime} 323 \%$} \\
\hline No ha mejorado & 8 & 0 & \\
\hline Mejora en la jerarquía & 0 & 6 & \multirow{2}{*}{$\mathrm{P}=0{ }^{\prime} 0054 \%$} \\
\hline No hay mejora & 12 & 0 & \\
\hline Han aumentado las diferenciaciones progresivas & 5 & 6 & \multirow{2}{*}{$P=2{ }^{\prime} 48 \%$} \\
\hline No las han aumentado & 7 & 0 & \\
\hline $\begin{array}{l}\text { Ha diferenciado progresivamente el concepto } \\
\text { desertificación }\end{array}$ & 1 & 5 & \multirow[t]{2}{*}{$\mathrm{P}=00^{\prime} 38 \%$} \\
\hline No lo ha diferenciado progresivamente & 11 & 1 & \\
\hline $\begin{array}{l}\text { Ha diferenciado progresivamente el concepto de } \\
\text { actividad humana }\end{array}$ & 1 & 5 & \multirow{2}{*}{$\mathrm{P}=0,38 \%$} \\
\hline No lo ha diferenciado progresivamente & 11 & 1 & \\
\hline $\begin{array}{l}\text { Las reconciliaciones integradoras han aumentado más } \\
\text { del doble }\end{array}$ & 6 & 5 & \multirow{2}{*}{$\mathrm{P}=2{ }^{\prime} 9 \%$} \\
\hline $\begin{array}{l}\text { Las reconciliaciones integradoras han aumentado } \\
\text { menos o no han aumentado }\end{array}$ & 6 & 1 & \\
\hline
\end{tabular}

En la primera columna se señalan las diferencias encontradas en el análisis de los MMCC posteriores de los alumnos. Se puede apreciar que estas diferencias en realidad hacen referencia a los indicadores de aprendizaje. Como es sabido, cuanto menor es la probabilidad de ocurrencia, mayor es la significatividad de la diferencia entre los grupos.

Tabla III

Comparación entre resultados de las entrevistas posteriores.

\begin{tabular}{|c|c|c|c|}
\hline & Grupo control & Grupo experimental & Probabilidad de ocurrencia \\
\hline Han disminuido el número de errores & 5 & 6 & \multirow{2}{*}{$\mathrm{P}=2{ }^{\prime} 4 \%$} \\
\hline No lo han disminuido & 7 & 0 & \\
\hline $\begin{array}{l}\text { Los alumnos relacionan la desertificación con la desertización, con } \\
\text { la pérdida de fertilidad, con la degradación del suelo, con la erosión } \\
\text { del suelo }\end{array}$ & 3 & 5 & \multirow[t]{2}{*}{$\mathrm{P}=3 \%$} \\
\hline No establecen semejantes relaciones & 9 & 1 & \\
\hline $\begin{array}{l}\text { Alumnos que establecen alguna relación con la desertificación y se } \\
\text { implican con ella }\end{array}$ & 5 & 6 & \multirow[t]{2}{*}{$\mathrm{P}=2^{\prime} 48 \%$} \\
\hline No establecen ninguna relación & 7 & 0 & \\
\hline $\begin{array}{l}\text { El alumno establece relaciones concretas y también plantea alterna- } \\
\text { tivas implicantes en el ámbito personal coherentes }\end{array}$ & 3 & 5 & \multirow[t]{2}{*}{$\mathrm{P}=3 \%$} \\
\hline No las establece & 9 & 1 & \\
\hline
\end{tabular}

En la primera columna se señalan las diferencias encontradas en el análisis proposicional realizado en las entrevistas posteriores de los alumnos. Se puede apreciar que estas diferencias en realidad hacen referencia a los indicadores de aprendizaje. Cuanto mayor es la probabilidad de ocurrencia, menor es la significatividad de la diferencia entre los grupos. 
Tabla IV-V

\section{Comparación entre fragmentos de las entrevistas realizadas a Mikel y E lisa antes y después de la instrucción.}

\begin{tabular}{|c|c|}
\hline MIKEL. TALDE ESPERIMENTALA & \\
\hline LEHENENGO ELKARRIZKETA & BIGARREN ELKARRIZKETA \\
\hline $\begin{array}{l}\text { - Onu, Fao eta antzeko munduko instituzioak, Europako Kontseiluak } \\
\text { eta Espainia Estatuko gobernu eta erakundeak diote, Lurra planetak } \\
\text { dituen arazoetatik larrienetako bat dela zonalde zabaletako } \\
\text { desertifikazioa. Ulertzen ahal duzu arazo honen esanahia? Zerekin } \\
\text { erlazionatzen duzu? } \\
\text { - Euria ez egitea eta lurra desertifikatzea eta... } \\
\text { - Baina zerekin erlazionatuko zenuke lurra desertifikatze hori? } \\
\text { - Pues zuhaitzak moztea, lurra biluzik geratzea. } \\
\text {... } \\
\text { - Pentsatzen duzu Nafarroan ba ote dago desertifikazioaren } \\
\text { arriskurik? } \\
\text { - Bai, adibidez Bardenatan eta erriberan... ia ari da... } \\
\text { - Nafarroako Ze informazio aztertu beharko zenuke ziurtatzeko } \\
\text { dagoen ala ez arrisku hori? } \\
\text { - Zenbat euri egiten duen, zenbat higadura dagoen eta zenbat ohian } \\
\text { dagoen ... } \\
\text {... } \\
\text { - Zer egingo zenuke zuk, desertifikazio arazoaren aurrean? } \\
\text { - Ezer, bueno ia desertifikatu behar dena uste dut ezin dela egin ezer. } \\
\text { Bueno ba oihanak eta hori ez moztu, zeren bestela izan behar ditugu } \\
\text { hamahiru... desertu munduan...eta ez dakit, pues galarazi behar dela } \\
\text { oihanak moztea, lurra hainbeste ez esplotatzea... } \\
\text { - Eta zuk egin dezakezuna? } \\
\text { - Nik? } \\
\text { - Bai. } \\
\text { - Pues nik uste dut ezin dudala ezer egin. Ah bueno bai, adibidez gu } \\
\text { pues ezin dugu kontsumitu hainbeste gauza, oihanetik datorrena... ez } \\
\text { dakit, zergatik horrela irabazten dute dirua eta mozten dute gehiago } \\
\text { oihanak...eta ur gutxiago, kontsumoa. }\end{array}$ & $\begin{array}{l}\text { - ONU, FAO eta antzeko munduko instituzioak, Europako } \\
\text { Kontseiluak eta Espainia Estatuko gobernu eta erakundeak diote, } \\
\text { Lurra planetak dituen arazoetatik larrienetako bat dela zonalde } \\
\text { zabaletako desertifikazioa. Ulertzen ahal duzu arazo honen esanahia? } \\
\text { - Zerekin erlazionatzen duzu? } \\
\text { - Zerekin? Ba orain, ez dakit, higadurarekin eta higadurak egiten duen } \\
\text { prozesua edo... sortzen duen arazoarekin. } \\
\text { - Ze arazo izango litzateke higadurak probokatzen duena? } \\
\text { - Ba, desertifikazioa... } \\
\text { - Konkrezioren bat emango bazenu, zerekin erlazionatuko zenuke? } \\
\text { (azalpen gehiago) } \\
\text { - Lurzoruaren higadura eta... } \\
\text { - Eta lurzoruaren higadura hori zerekin erlazionatzen duzu? Ze } \\
\text { problematikarekin? } \\
\text { - Ba azkenean lurzorua antzu geratu behar dela eta lurzorutik hazten } \\
\text { da guztia ez... hazten da bizitza ... bueno lurzorua eta ura ez? Hazten } \\
\text { dira zuhaitzak eta ... } \\
\text {... } \\
\text { - Nafarroan ba ote dago desertifikazioaren arriskurik? } \\
\text { - Bai. } \\
\text { - Zertan fijatuko zinateke baiezko hori esateko? } \\
\text { - Zertan? Adibidez, malda baldin badago, eta landaretza gutxi eta ... } \\
\text { - Orduan zuk begiratuko zenuke Nafarroako malda edo erliebea, } \\
\text { begiratuko zenuke landaretza nola dagoen, zer gehiago? } \\
\text { - Ea malda duen, ea landaretza duen, zenbat ur erortzen den edo nola } \\
\text { erortzen den ura, oso gogor edo... eta haizea dagoen edo... } \\
\text {-... } \\
\text { - Norrela... } \\
\text { - Eta orduan zer proposatuko zenuke zuk, desertifikazioa arazoaren } \\
\text { aurrean? } \\
\text { - Pues orokorrean kontzientziatze kanpaina bat edo... } \\
\text { - Kanpaina horretan zein izango litzateke mezu inportanteena? } \\
\text { - Pues, etorkizunerako oso gaizki eduki behar ditugula gauzak eta } \\
\text { azkenean ezingo dela bizi planetan ... } \\
\text { positiboak egingo zenituzke? } \\
\text { - }\end{array}$ \\
\hline
\end{tabular}




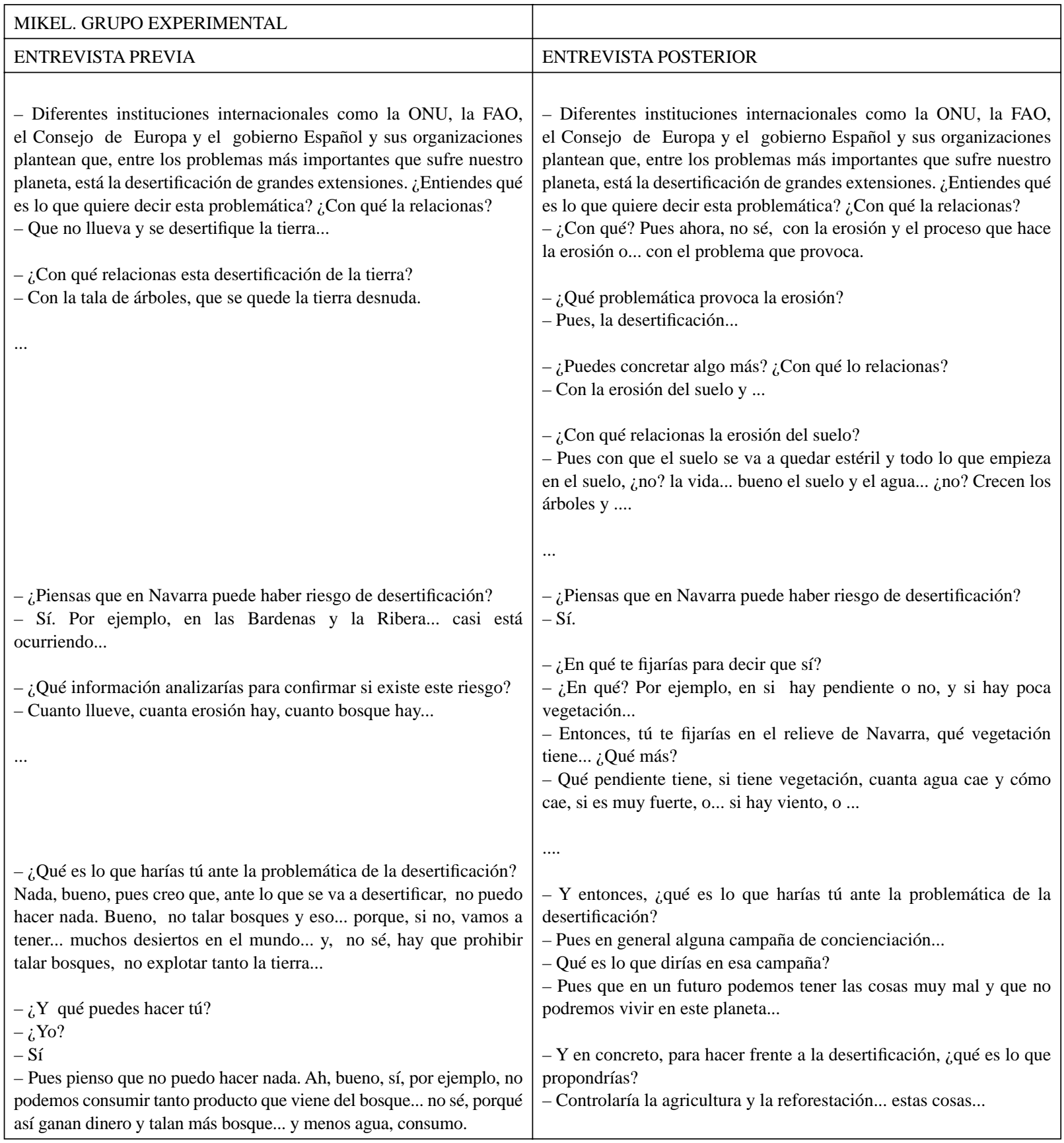


Tabla V

\begin{tabular}{|c|c|}
\hline ELISA. KONTROL TALDEA & \\
\hline LEHENENGO ELKARRIZKETA & BIGARREN ELKARRIZKETA \\
\hline $\begin{array}{l}\text { ONU, FAO eta antzeko munduko instituzioak, Europako Kontseiluak } \\
\text { eta Espainia Estatuko gobernu eta erakundeak ekologista taldeekin } \\
\text { batera diote, Lurra planetak dituen arazoetatik larrienetako bat dela } \\
\text { zonalde zabaletako desertifikazioa. Ulertzen ahal duzu arazo honen } \\
\text { esanahia? Zerekin erlazionatzen duzu? } \\
\text { Baietz uste dut. } \\
\text { Zerekin erlazionatzen duzu? } \\
\text { Kapa de ozonoarekin, ... } \\
\text { Zer izango litzatekeen desertifikazioa edo zer bururatzen zaizu dela? } \\
\text { Eh? lurrazalean dauden landareak desagertzea, ba, } \\
\text {... } \\
\text { Nafarroan ba ote dago desertifikazioaren arriskurik? } \\
\text { Orain ez baina urteak pasa eta horrela jarraitzen bada bai. } \\
\text { Zer aztertu beharko zenuke halako galdera erantzuteko? } \\
\text { Ba ondoko lurraldeetan gertatzen hasten bada, ba denbora pasatzen } \\
\text { den heinean Nafarrora iritxiko da. } \\
\text { Inguruan zer gertatzen ari den galdetuko zenuke. Beste zerbait? } \\
\text {... } \\
\text { Zer egingo zenuke zuk, desertifikazioa arazoaren aurrean? } \\
\text { Bakarrik, lantegien lan egiteko orduak murriztea eta autoak ahal } \\
\text { bezain gutxi hartzea, bakarrik beharrezkoak direnean. Zuhaitzak } \\
\text { landatzea, ... ez dakit. }\end{array}$ & $\begin{array}{l}\text { ONU, FAO eta antzeko munduko instituzioak, Europako Kontseiluak } \\
\text { eta Espainia Estatuko gobernu eta erakundeak, ekologista taldeekin } \\
\text { batera diote, Lurra planetak dituen arazoetatik larrienetako bat dela } \\
\text { zonalde zabaletako desertifikazioa. Ulertzen ahal duzu arazo honen } \\
\text { esanahia? Zerekin erlazionatzen duzu? } \\
\text { Bai. Ba zuhaitzen desagerpena, bueno, landaretzaren desagerpena, } \\
\text { eta ... } \\
\text { Eta horren ondorioz, ondorio problematikoren bat edo ikusten duzu? } \\
\text { Bai, ba landaretza ez dagoen lekua ba animaliak ere ez, eta pixkanaka } \\
\text { pixkanaka lurralde gehiago hartzen joango dela desertuak osea. } \\
\text {... } \\
\text { Nafarroan ba ote dago desertifikazioaren arriskurik? } \\
\text { Bai, denbora pasata bai. } \\
\text { Zer aztertu beharko zenuke halako galdera erantzuteko? } \\
\text { Ba, ea non dagoen desertifikazioa gertatzen ari den lekuren bat eta } \\
\text { ikusteko ea denborarekin irits daitekeen edo ez. } \\
\ldots \\
\text { Zer egingo zenuke zuk, desertifikazioa arazoaren aurrean? } \\
\text { Gehiago zaindu dezaket... osea pentsatzea nik egiten dudan } \\
\text { bizitzarekin zer egiten dudan hori, osea desertifikazioa... } \\
\text { Adibidez zuk egiten duzu zerbait areagotu dezakeena? } \\
\text { Pues bai, eh, egin dezaket zuhaitzak landatu eta .. } \\
\text { Zuk ikusten duzu zuren esku badagoela zerbait egitea. adibidez } \\
\text { zuhaitzak landatzea. ... }\end{array}$ \\
\hline
\end{tabular}


Tabla V

\begin{tabular}{|c|c|}
\hline & \\
\hline ENTREVISTA PREVIA & ENTREVISTA POSTERIOR \\
\hline $\begin{array}{l}\text { - Diferentes instituciones internacionales como la ONU, la FAO, } \\
\text { el Consejo de Europa y el gobierno Español y sus organizaciones } \\
\text { plantean que, entre los problemas más importantes que sufre nuestro } \\
\text { planeta, está la desertificación de grandes extensiones. ¿Entiendes qué } \\
\text { es lo que quiere decir esta problemática? ¿Con qué la relacionas? } \\
\text { - Creo que sí. } \\
\text { - Con qué lo relacionas? } \\
\text { - Con la capa de ozono... } \\
\text { - ¿Qué se te ocurre decir sobre la desertificación? } \\
\text { - ¿Eh? La desaparición de la vegetación de la superficie terrestre. } \\
\ldots \\
\text {-..Piensas que en Navarra puede haber riesgo de desertificación? } \\
\text { - Ahora no, pero cuando pasen los años, si sigue así, sí. } \\
\text { - ¿Qué es lo que analizarías para contestar esta pregunta? } \\
\text { - Pues si empieza a ocurrir en los territorios que tenemos al lado, pues } \\
\text { con el tiempo puede llegar a Navarra. } \\
\text {.... }\end{array}$ & $\begin{array}{l}\text { - Diferentes instituciones internacionales como la ONU, la FAO, } \\
\text { el Consejo de Europa y el gobierno Español y sus organizaciones } \\
\text { plantean que, entre los problemas más importantes que sufre nuestro } \\
\text { planeta, está la desertificación de grandes extensiones. ¿Entiendes qué } \\
\text { es lo que quiere decir esta problemática? ¿Con qué la relacionas? } \\
\text { - Pues con la desaparición de árboles, bueno, la desaparición de } \\
\text { vegetación. } \\
\text { - ¿Relacionas alguna problemática con estos hechos? } \\
\text { - Sí, pues donde no hay vegetación tampoco hay animales, y que } \\
\text { poco a poco el desierto va a ir cogiendo más territorio... } \\
\text {... } \\
\text { - ¿Piensas que en Navarra puede haber riesgo de desertificación? } \\
\text { - Sí, cuando pase el tiempo sí. } \\
\text { - ¿Qué es lo que analizarías para contestar esta pregunta? } \\
\text { - Pues, dónde está ocurriendo hoy la desertificación, para ver si con } \\
\text { el tiempo puede llegar o no. } \\
\text {... } \\
\text { - ¿Qué puedes hacer ante la problemática de la desertificación? } \\
\text { - Puedo cuidar más... es decir pensar que, con lo que hago en mi } \\
\text { vida, es decir, la desertificación... } \\
\text { - Por ejemplo, haces algo que pueda intensificar la desertificación? } \\
\text { - Pues sí, puedo plantar árboles, y... } \\
\text { - Tú ves que puedes hacer algo en contra de la desertificación como } \\
\text { lantarboles. }\end{array}$ \\
\hline
\end{tabular}




\section{A MODO DE CONCLUSIÓN}

Los resultados obtenidos del análisis comparativo de los MMCC y de las transcripciones de las entrevistas, tanto con relación al registro anterior y posterior a la instrucción como con relación al contraste grupo experimental y control, nos indican unas diferencias significativas con relación a aspectos importantes del aprendizaje del alumnado. En los MMCC realizados por el alumnado perteneciente al grupo experimental encontramos que, con una probabilidad de ocurrencia menor al 3\%, se identifican unas diferencias con respecto al alumnado del grupo control, que consideramos evidencias de un aprendizaje más significativo. A este respecto es importante señalar la existencia de una disminución en el número de errores, de una mejora en la jerarquía establecida entre los conceptos, un aumento del número de diferenciaciones progresivas de los conceptos más inclusivos y, además, un incremento del número de reconciliaciones integradoras. En consonancia con estos datos, el análisis proposicional de las entrevistas expresa claramente que el alumnado del grupo experimental también tiene menos ideas erróneas, incorpora nuevos conceptos a la hora de dar significado a la desertificación y es capaz de proponer respuestas más implicantes en relación con la misma.

Consideramos que el alumnado del grupo experimental, al manejar materiales instruccionales conceptualmente más transparentes, ha podido identificar mejor las ideas más inclusivas y globales sobre la problemática tratada, diferenciando progresivamente, con mayor eficacia, unas actividades humanas y unos procesos climáticos que, a través de las pertinentes reconciliaciones integradoras, han dado un significado más elaborado a la problemática de la desertificación. Además, estos mismos alumnos y alumnas, a lo largo de la instrucción, han tenido la oportunidad de acercarse a esta problemática con una implicación personal más clara y crítica. Si bien es cierto que el tipo de investigación que aquí se ha presentado no contiene todos los elementos necesarios para poder generalizar los resultados obtenidos, es importante, en nuestra opinión, señalar que, en un contexto escolar convencional, el hecho de implementar un módulo instruccional fundamentado teóricamente ha posibilitado que un grupo de alumnos haya tenido la oportunidad de aprender, con relación a una problemática medioambiental como la desertificación, de forma más significativa que en unas condiciones más tradicionales.

Por todo ello, queremos resaltar, finalmente, la virtualidad del marco teórico de Ausubel, Novak y Gowin y del uso de las herramientas cognitivas emanadas del mismo (MC y diagrama u ve) para facilitar un aprendizaje más significativo de los alumnos y alumnas y para inducir un cambio de actitud favorable en relación con la problemática medioambiental presentada.

\section{REFERENCIAS BIBLIOGRÁFICAS}

BREITING, S. (1997). Hacia un nuevo concepto de educación ambiental, en Carpeta informativa, pp. 81-89. Balsaín: CENEAM.

BRUMSTED, C. (1990). «The Degree of Student Engagement in Meaningful Learning Using Cooperative Learning and Student-Selected Subject Matter in Environmental Studies». Tesis no publicada. Ithaca, Nueva York: Cornell University, Department of Education.

CALVO, F. (1994). Estadística aplicada. Bilbao: Deusto, D L.

CAMPBELL, D. y STANLEY, J. (1970). Diseños experimentales y cuasi experimentales. Amorrortu: Buenos Aires.

ERIC (1992-2002/12). Educational Resources Information Center. [Base de datos bibliográfica en CD-ROM]. Norwood, MA: Silver Platter International.

GARCÍA, J. E. (1995). La transición desde el pensamiento simple hacia un pensamiento complejo en la construcción del conocimiento escolar. Investigación en la escuela, 27, pp. 7-20.
GARCÍA, J. E. (1998). Problemas y líneas de investigación en educación ambiental: el caso del proyecto IRES. Grupo de trabajo: investigación y evaluación en educación ambiental. III Jornadas de Educación Ambiental. Pamplona. Sin publicar.

POZO, J.L. y GÓMEZ CRESPO, M.A. (1998). Aprender y enseñar ciencia. Madrid: Morata.

GONZÁLEZ, F. M. y IRAIZOZ, N. (1994). Los mapas conceptuales. Un ejemplo de aplicación en el diseño curricular e instruccional en ciencias. La Escuela en Acción, 4, pp. 13-18.

GONZÁLEZ, F. M. y IRAIZOZ, N. (1995). Errores conceptuales en alumnos de enseñanza primaria: implicaciones educativas. Estudios de Pedagogía y Psicología, 7, pp. 67-116.

GONZÁLEZ, F. M. y IRAIZOZ, N. (1996-97): Diagnosis de errores conceptuales en ciencias y reflexiones para el cambio conceptual. Huarte de San Juan, 2-3, pp. 57-90. Facultad de Humanidades y Ciencias Sociales de la Universidad Pública de Navarra. 
GONZÁLEZ, F. M. y IRAIZOZ, N. (2001). Los mapas conceptuales y el aprendizaje significativo. Alambique, 28, pp. 39-51.

GONZÁLEZ, F. M., MORÓN, C., NOVAK, J. D. (2001). Errores conceptuales. Diagnosis, tratamiento, y reflexiones. Pamplona: Eunate.

GONZÁLEZ, F. M. y NOVAK, J. D. (1993). Aprendizaje significativo. Técnicas y aplicaciones. Buenos Aires: Cincel- Kapelusz.

GONZÁLEZ, F. M. y NOVAK, J. D. (1996). Aprendizaje significativo. Técnicas y aplicaciones (2a ed.). Madrid: Ediciones Pedagógicas.

GOWIN, D. (1981). Educating. Nueva York: Ithaca. Cornell University Press.

GURUCEAGA, A. (1994). Ingurugiroaren arazo baten trataera didakto konstruktibista: desertifikazioa eta uholdeak. Tantak,12, pp. 77-107.

GURUCEAGA, A. (2001). «Ikaskuntza esanguratsua eta ingurugiro hezkuntza». («Aprendizaje significativo y educación ambiental»). Tesis doctoral. Nafarroako Unibertsitate Publikoa (Universidad Pública de Navarra).

LEAP Project (Learning about Ecology, Animals and Plants) (1995). College of Agriculture and Life Sciences at Cornell University.

LUCAS, A.M. (1980). Science and environmental education: Pious hopes, self praise and disciplinary chauvinism. Studies in Science Education, 7, pp. 1-26.

LUCKO, B., DISSINGER, J. y ROTH, R. (1982). Evaluation of Environmental Education Programs at the Elementary and Secondary School Lerels. Journal of environmental Education, 14 (4), pp. 7-12.

MOREIRA, M. A. (1997). Aprendizajem significativa: um Conceito Subyacente. Encuentro Internacional sobre Aprendizaje Significativo. Burgos: Servicio de Publicaciones de la Universidad de Burgos.

MOREIRA, M. A. y DOMÍNGUEZ, M. E. (1989). A entrevista clinica como técnica de pesquisa em ensino. Manuscrito sin publicar.
NOVAK, J. D. (1978). A Theory of Educaction as a Basis for Environmental Education. Bakshi, T. S. y Naveh, Z. (eds.). Environmental Education. Principles, Methods and applications, pp. 129-138. Nueva York: Plenum Press.

NOVAK, J. D. (1988). Constructivismo humano: un consenso emergente. Enseñanza de las Ciencias, 6 (3), pp. 213-223.

NOVAK, J. D. (1997). Clarify with Concept Maps Revisited. Actas del Encuentro Internacional sobre Aprendizaje Significativo, pp. 47-63. Burgos: Servicio de Publicaciones de la Universidad de Burgos.

NOVAK, J. D. y GOWIN, D. (1988), Aprendiendo a aprender. Barcelona: Martínez Roca.

NOVO, M. (1998). La educación ambiental. Bases éticas, conceptuales y metodológicas. Madrid: Ediciones Unesco y Editorial Universitas, SA.

PINES, L. (1979). Program in Psychology and Foundations of Education. University of Maine. Georgia.

PINES, L., NOVAK, J. D., POSNER, G. J. y VANKIRK, J. (1978). The clinical interview: A method for evaluating cognitive structure. Research report núm. 6. Cornell Universyty, Ithaca, NY.

POZUETA, E. (1994). Ingurugiroari buruzko gai baten irakaskuntzarako ikuspuntu konstruktibista bat: janari-ontzien koste ekologikoa. Tantak, 12, pp. 109-137.

RICKINSON, M. (2001). Learners and Learning in Environmental Education. Environmental Education Research, 7(3), pp. $207-320$

STILWELL, S. y BRODY, M. (1987). Cross Cultural Analysis of Student Understanding of Marine Science and Natural resource Concepts Related to the Gulf of Maine. Proceedings of the Second International Seminar on Misconceptions and Educational Strategies in Science and Mathematics. Cornell University, Ithaca, NY. Vol. II, pp. 490-498.

TVE (1995). «Cita con la tierra», programa emitido el 9 de diciembre de 1995 con el título Desertificación. 
Figura 1

Mapa conceptual sobre la desertificación (G uruceaga, 1996).

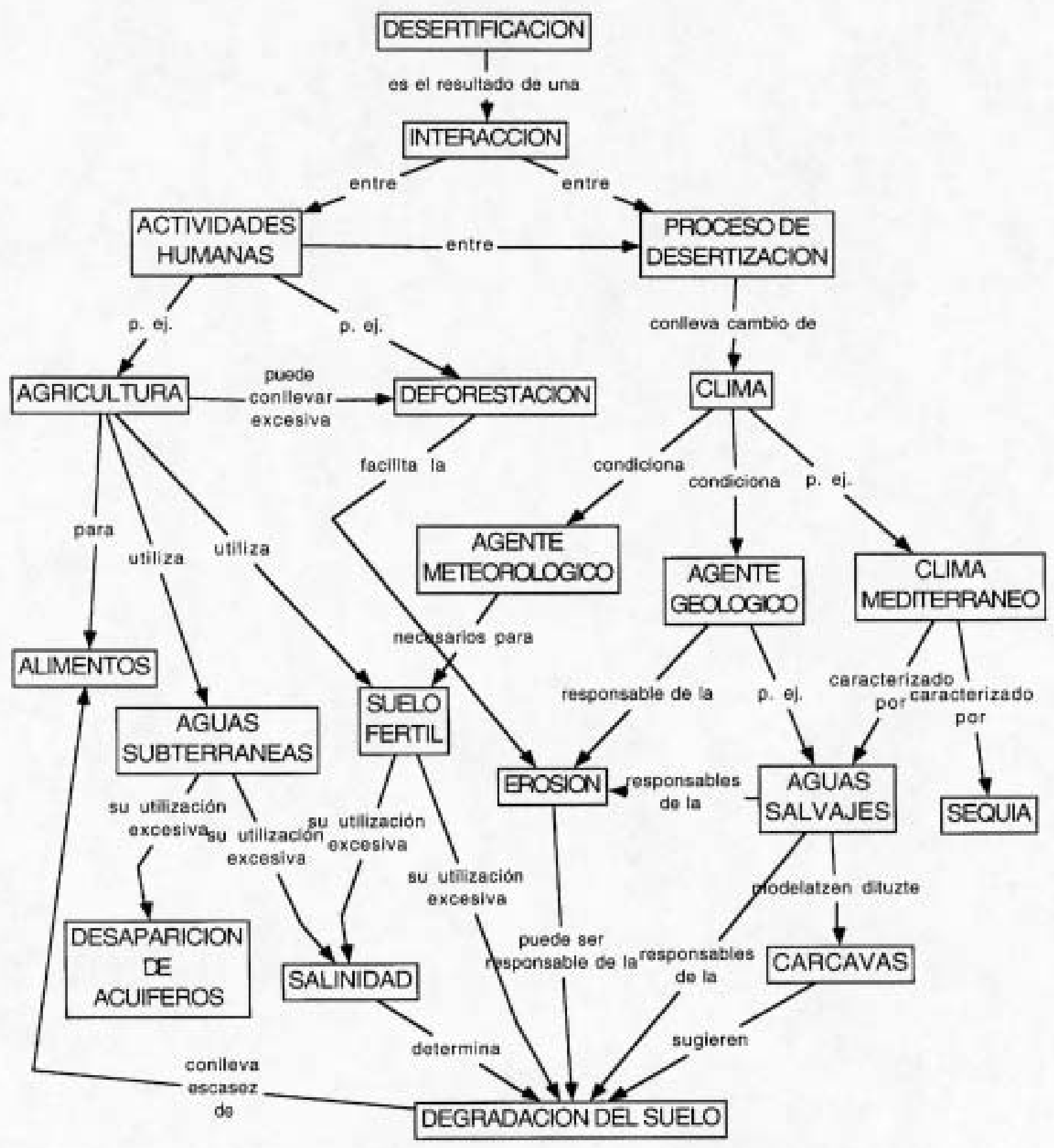




\section{Natur aisntrink}

\section{EZAGUTU DEZAGIN INGURUGIROKO ARAZO BAT}

tzena Miksh

Gela:, 录每

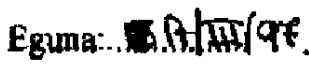

A0 Egin dezagun konzeptu aurre mapa hau:

akuiferoak edo luapeko urak

dkuifercen desagerpena

- basamorturatze prozesua

deforestuzion

descrtifikazioa

elikagaial

rragile meteorologikoak gazipena

- giza ekintrak

higadura

interalzzioa
- Kanpoeragile geologikoak

7 karkabak / kole solu nat de-

Jkhima

lehortes

hurzoru emankorta

luzonuaren degratazioa

mediterranear ldims

nelcezaritza

ur- basatiak

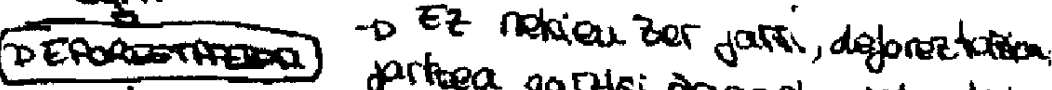
Góns prtwea gartki dasoola usto dut alcaratar.

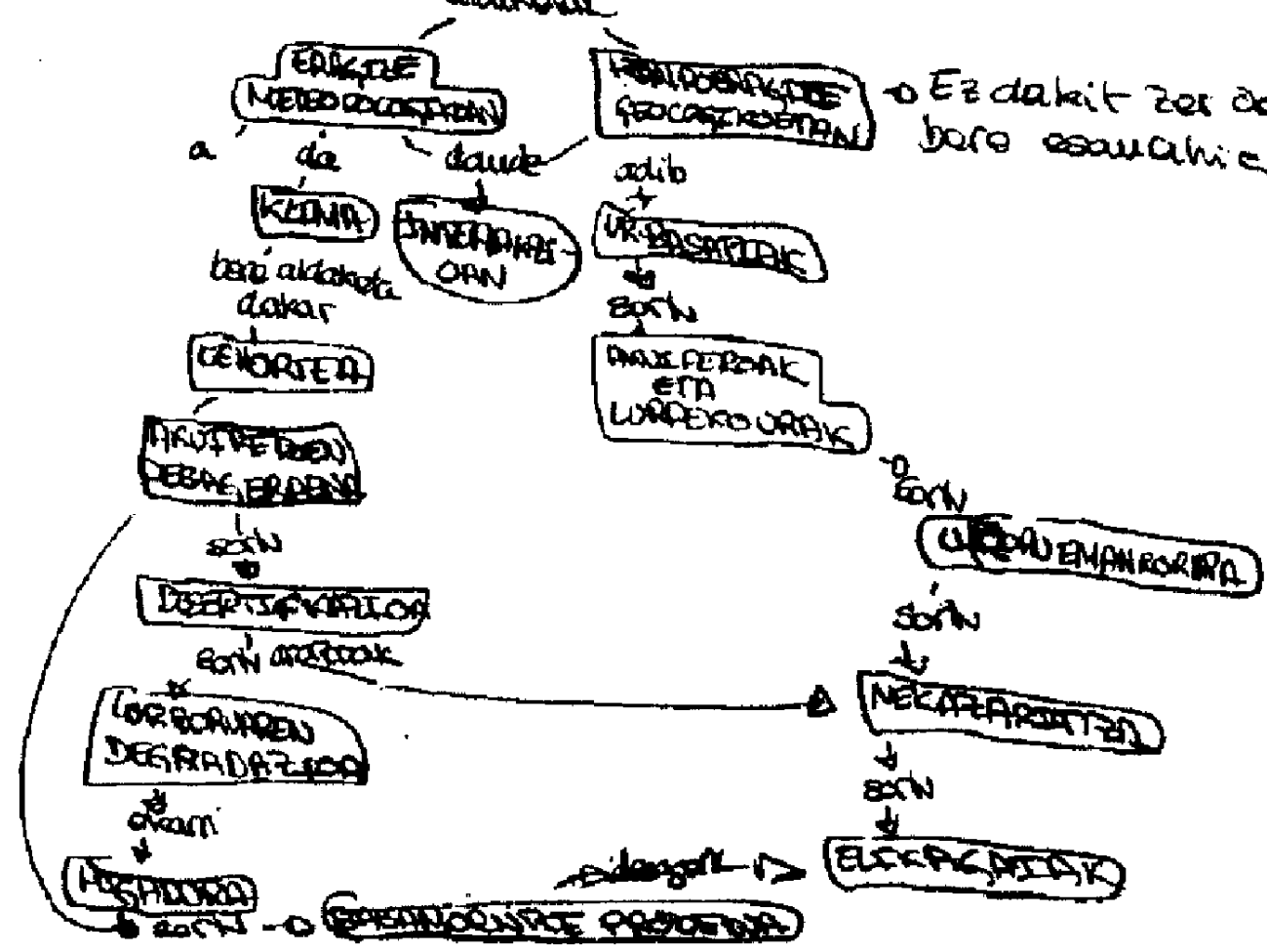


INVESTIGACIÓN DIDÁCTICA

Figura 2

Mapa conceptual previo de Mikel.

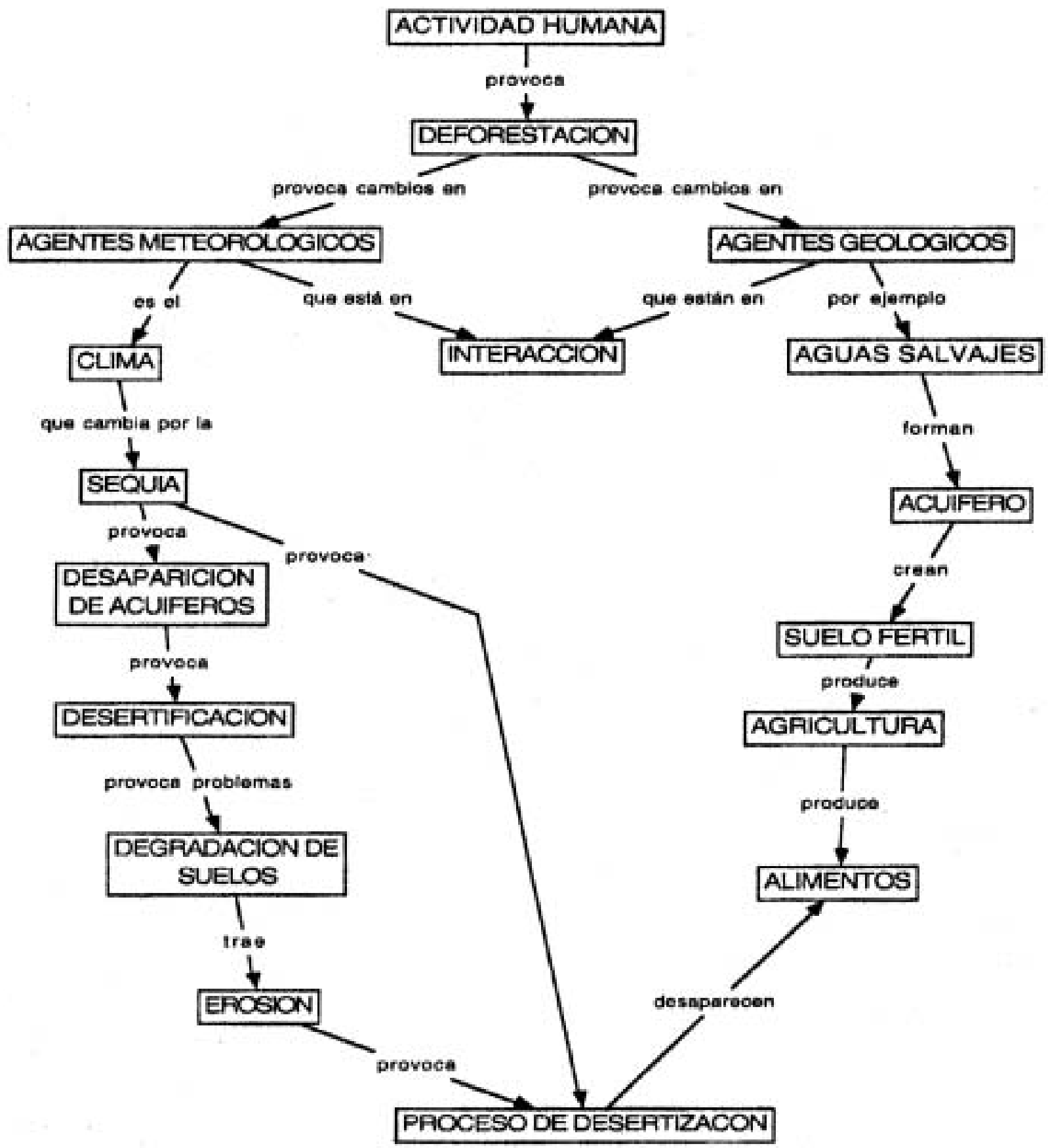




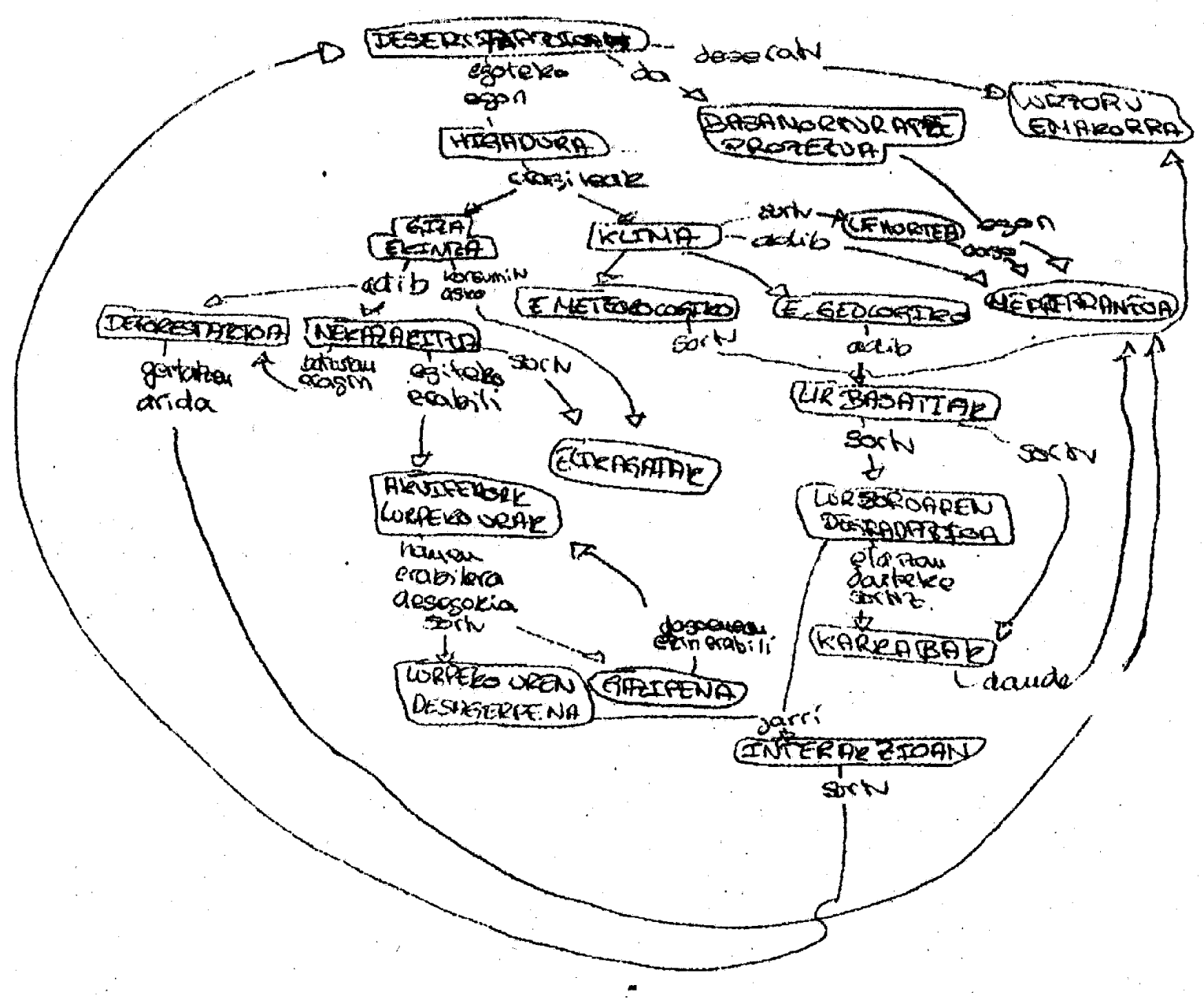

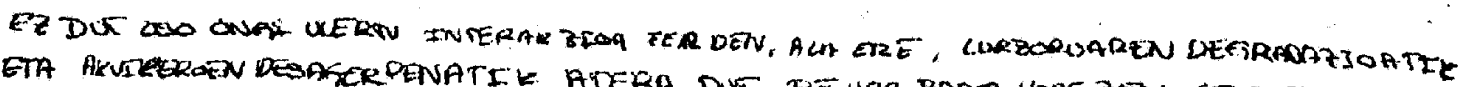
ETA SIIMBRTE ATEPACER. 
Figura 3

Mapa posterior de Mikel.

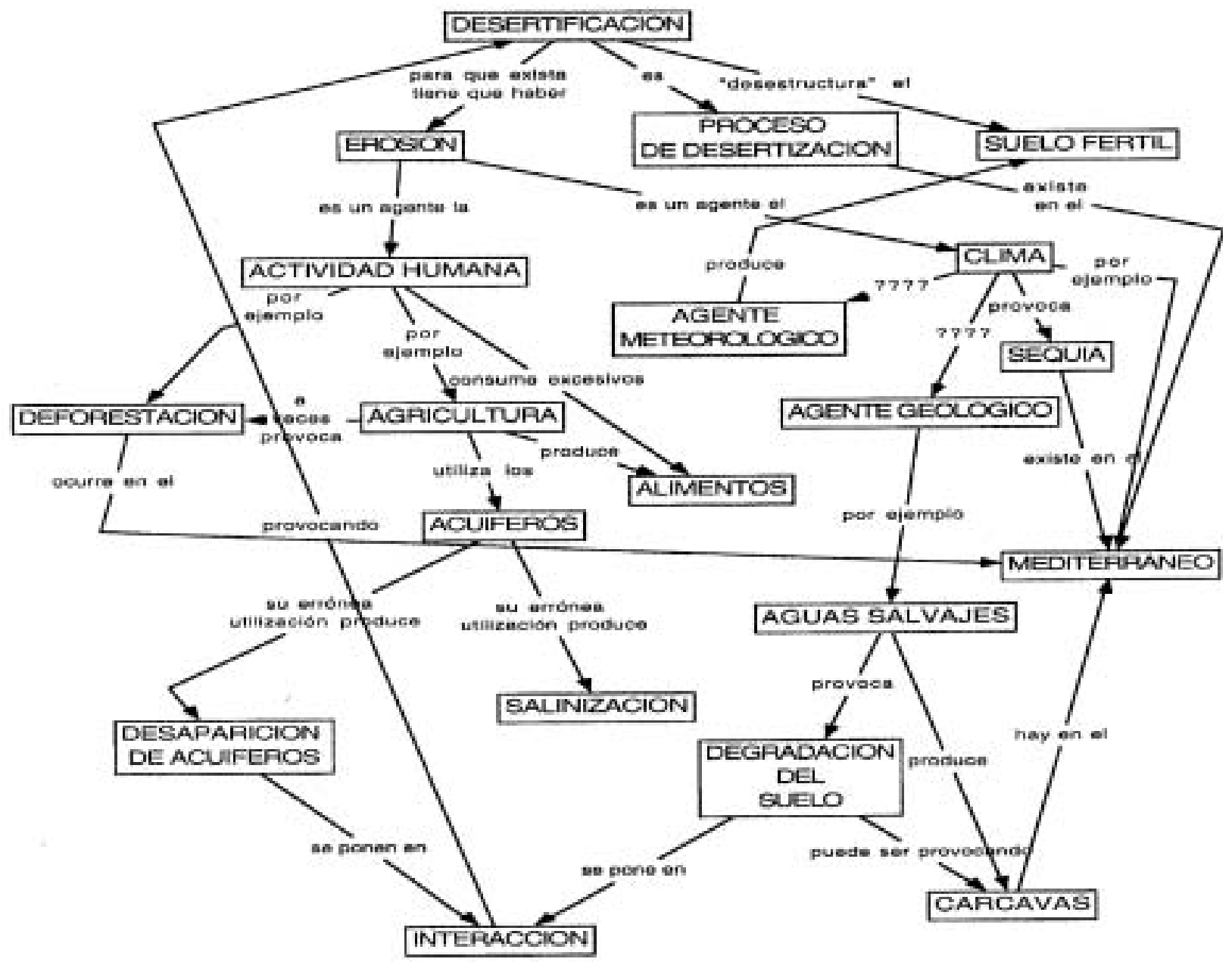




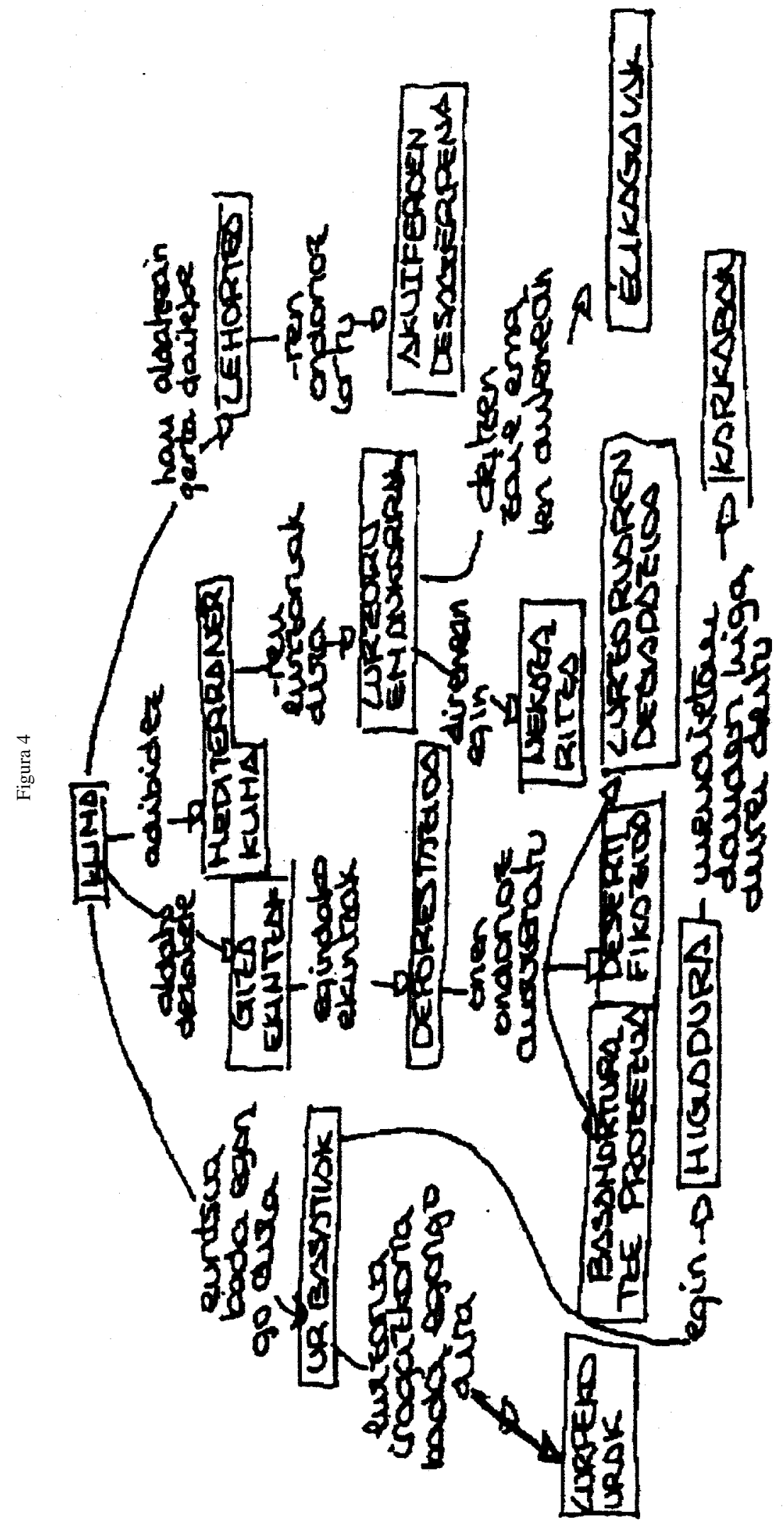




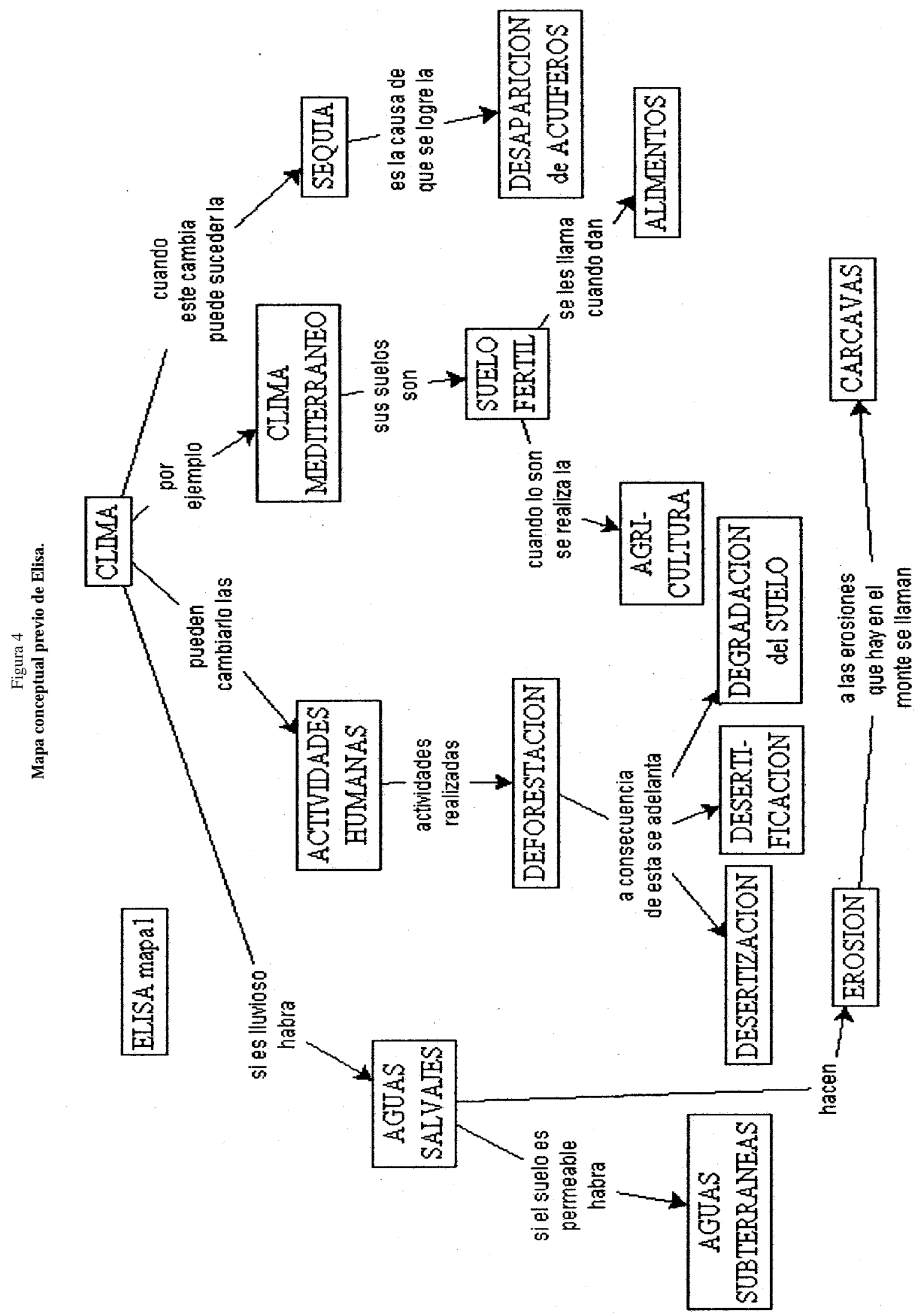




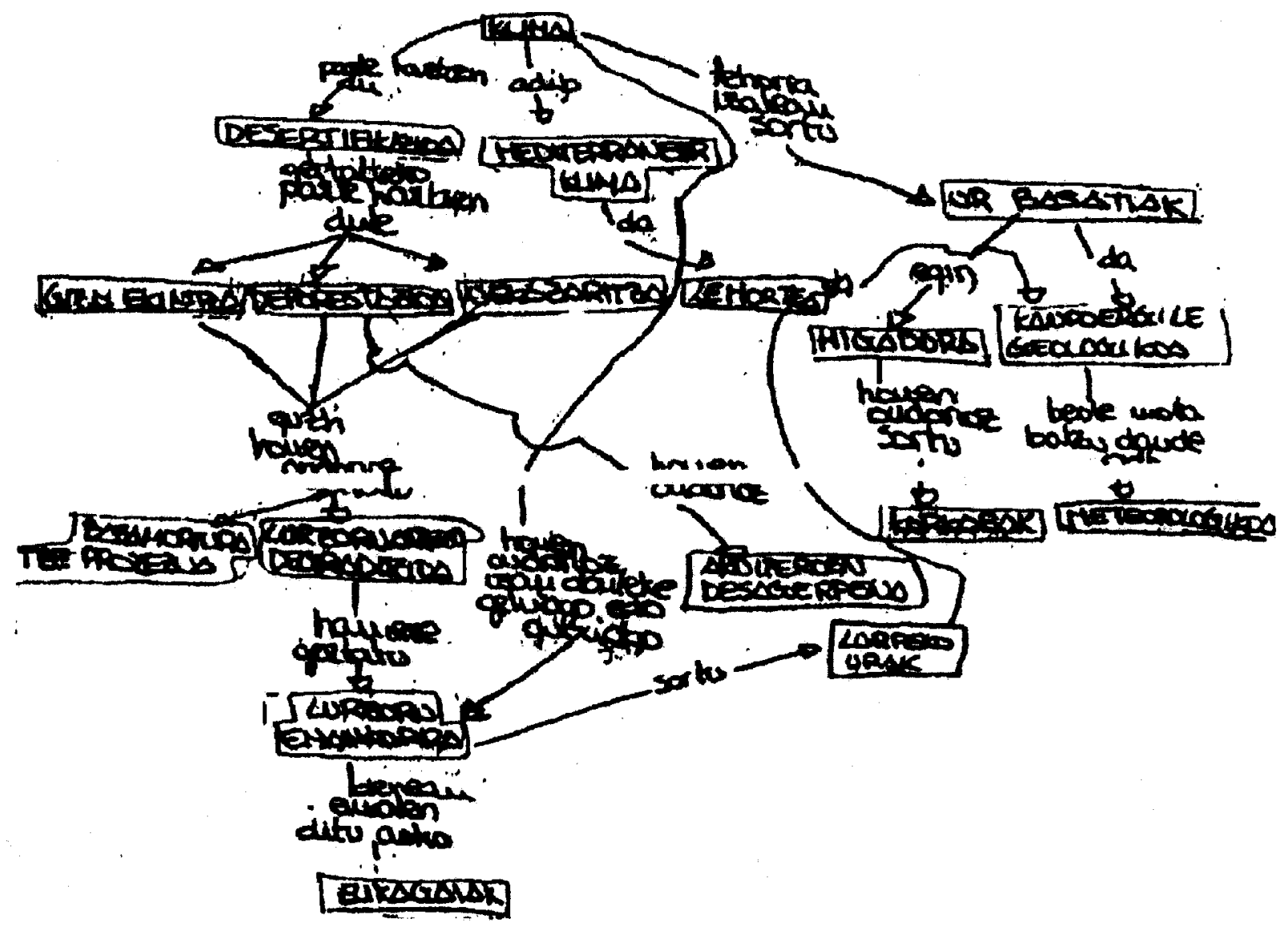


Figura 5

Mapa conceptual posterior de E lisa

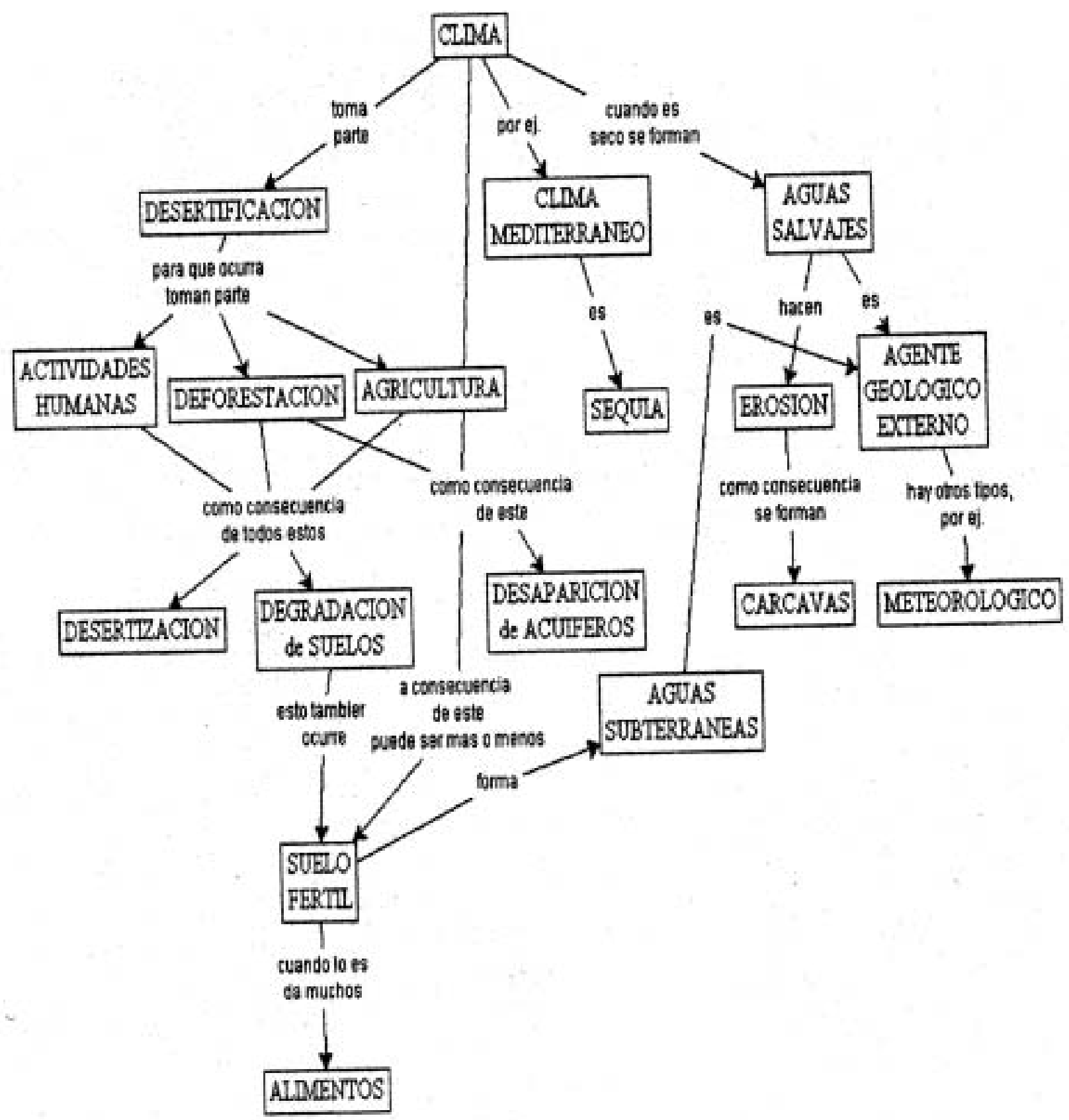

